\title{
O Uso da Prospecção como Ferramenta na Busca de Tecnologias de Gerenciamento de Resíduos Sólidos no Brasil
}

\author{
The Use of Prospecting as a Tool in Search for Management \\ Technologies Solid Waste in Brazil
}

\author{
Laudilene Macedo Bispo ${ }^{1}$ \\ Marcelo Soares Teles Santos ${ }^{2}$ \\ Josiele Ferreira do Nascimento ${ }^{1}$ \\ ${ }^{1}$ Universidade Estadual de Santa Cruz, Ilhéus, BA, Brasil \\ ${ }^{2}$ Universidade Federal do Sul da Bahia, Itabuna, BA, Brasil
}

\begin{abstract}
Resumo
Este trabalho teve como objetivo principal utilizar a prospecção tecnológica de patentes concedidas no âmbito nacional para conhecer as inovações no segmento de Gerenciamento de Resíduos Sólidos (GRS) no Brasil, em especial as etapas de não geração, redução e reutilização, destacadas como prioritárias ao gerenciamento, segundo a Lei $n^{\circ}$ 12.305/2010, da Política Nacional de Resíduos Sólidos. A busca foi realizada na base de dados do Instituto Nacional de Propriedade Industrial (INPI), por meio da International Patent Classification (IPC) com os códigos de classificação "B09B3/00 (Destruição de lixo sólido ou transformação em algo útil ou inofensivo)", "C08J11/00 (Recuperação ou reaproveitamento de materiais residuais)". Como resultados da prospecção, foram encontradas 577 patentes, sendo que, apenas 27 patentes $(4,7 \%)$ foram relacionadas com as três primeiras prioridades do GRS. As novas regulamentações exigidas para as atividades relacionadas com resíduos sólidos estimularam ações em educação ambiental e investimentos em tecnologia e inovação no setor.
\end{abstract}

Palavras-chave: Prospecção. Patentes. Resíduos Sólidos. Lei 12.305/2010.

\begin{abstract}
The main objective of this work was to use the technological prospection of patents granted at the national level to know the innovations in the Solid Waste Management (GRS) segment in Brazil, especially the stages of non-generation, reduction and reuse, highlighted as priorities for management, according to Law No. 12,305 / 2010, of the National Policy on Solid Waste. The search was done in the database of the National Institute of Industrial Property (INPI), through the International Patent Classification (IPC) with the classification codes "B09B3 / 00 (Destruction of solid waste or transformation into something useful or harmless) "C08J11 / 00 (Recovery or reuse of waste materials)". As a result of the survey, 577 patents were found, with only 27 patents $(4.7 \%)$ related to the first three GRS priorities. The new regulations required for activities related to solid waste stimulated actions in environmental education and investments in technology and innovation in the sector.
\end{abstract}

Keywords: Prospecting. Patents. Solid Waste. Law 12.305/2010.

Área Tecnológica: Prospecção tecnológica. Inovação. 


\section{Introdução}

O avanço da ciência proporcionou grandes feitos para a humanidade, como a queda da mortalidade, o aumento da expectativa de vida e, consequentemente, a explosão demográfica. Como resultado dessa engrenagem, surgiram novas práticas de consumo e uso dos recursos naturais que, devido à falta de planejamento, ocorreram de forma predatória, gerando grandes quantidades de resíduos lançados na natureza sem o tratamento adequado.

Atualmente, no Brasil, mais de 63\% dos municípios despejam o seu lixo em lixões a céu aberto, causando danos ao ambiente e à saúde dos que vivem em seu entorno. Nesse contexto, nas últimas décadas ocorreram e tem ocorrido uma série de discussões relacionadas aos problemas ambientais decorrentes da inadequada gestão de resíduos sólidos no Brasil, o que levou à criação de leis ambientais específicas para o setor, como a Lei 9.795, de 27 de abril de 1999 (BRASIL, 1999), da Política Nacional de Educação Ambiental, e a Lei n 12.305, de 2 de agosto de 2010 (BRASIL, 2010), da Política Nacional de Resíduos Sólidos.

A Lei $n^{\circ}$ 12.305/2010, além de instituir a Política Nacional de Resíduos Sólidos, abordou diretrizes relativas à Gestão Integrada e ao Gerenciamento de Resíduos Sólidos no Brasil. O artigo $9^{\circ}$ dessa lei aponta a ordem de prioridade na gestão e no gerenciamento de resíduos sólidos: não geração, redução, reutilização, reciclagem, tratamento dos resíduos sólidos e disposição final ambientalmente adequada dos rejeitos. Essas prioridades refletem uma tendência mundial de não geração de resíduos sólidos, ou seja, atenção especial às atividades de não geração, redução e reutilização de resíduos sólidos, fazendo com que os resíduos, caso gerados, sejam reutilizados sem necessidade de nenhum tipo de processamento ou tratamento.

No entanto, o atendimento a essas prioridades requer, além de ações de capacitação ambiental com foco no consumo responsável, o desenvolvimento tecnológico e a inovação no setor na tentativa de alcançar alternativas de enfrentamento do problema do gerenciamento $e$ permitir o desenvolvimento sustentável. Nesse contexto, o presente trabalho tem como objetivo geral realizar prospecção tecnológica para avaliar a situação do desenvolvimento tecnológico e da inovação no setor de Gestão e Gerenciamento de Resíduos Sólidos no Brasil, em especial às atividades prioritárias de não geração, redução e reutilização.

Para alcançar o objetivo do trabalho, foi realizada uma busca na base de dados do Instituto Nacional de Propriedade Industrial do Brasil (INPI) em função do grupo temático de Gerenciamento de Resíduos (INPI, 2017), por meio dos códigos International Patent Classification (IPC) com os códigos de classificação "B09B3/00 (Destruição de lixo sólido ou transformação em algo útil ou inofensivo)" e "C08J11/00 (Recuperação ou reaproveitamento de materiais residuais)" que possui uma subdivisão com possíveis tecnologias relacionadas ao tema. Na mineração dos dados, os resultados obtidos foram agrupados de acordo com as atividades de interesse do artigo.

\section{Revisão de Literatura}

\subsection{Gestão Integrada e Gerenciamento de Resíduos no Brasil}

A Lei n ${ }^{\circ}$ 12.305, de 2 de agosto de 2010, institui a Política Nacional de Resíduos Sólidos e as diretrizes relativas à Gestão Integrada e ao Gerenciamento de Resíduos Sólidos. O artigo 4 dessa lei define a Política Nacional de Resíduos Sólidos, da seguinte forma (BRASIL, 2010): 
A Política Nacional de Resíduos Sólidos reúne o conjunto de princípios, objetivos, instrumentos, diretrizes, metas e ações adotados pelo Governo Federal, isoladamente ou em regime de cooperação com Estados, Distrito Federal, Municípios ou particulares, com vistas à gestão integrada $e$ ao gerenciamento ambientalmente adequado dos resíduos sólidos.

A respeito da gestão integrada de resíduos sólidos, o BNDES (2013, p. 35) afirma que a gestão dos serviços de limpeza pública e o manejo dos resíduos sólidos devem ser "[...] pensados e implementados por meio dos denominados 'arranjos institucionais', na sua forma mais adequada para a aplicabilidade e sustentabilidade das tecnologias.", e que pela sua configuração de atuação, esses podem ser dirigidos de maneira direta ou indireta, ou seja, em articulação com diversos atores e entidades.

Para os efeitos dessa Lei, o artigo 3 estabelece as seguintes definições (BRASIL, 2010):

Inciso X - Gerenciamento de Resíduos Sólidos: conjunto de ações exercidas, direta ou indiretamente, nas etapas de coleta, transporte, transbordo, tratamento e destinação final ambientalmente adequada dos resíduos sólidos e disposição final ambientalmente adequada dos rejeitos, de acordo com plano municipal de gestão integrada de resíduos sólidos ou com plano de gerenciamento de resíduos sólidos, exigidos na forma desta Lei; Inciso XI - Gestão Integrada de Resíduos Sólidos: conjunto de ações voltadas para a busca de soluções para os resíduos sólidos, de forma a considerar as dimensões política, econômica, ambiental, cultural e social, com controle social e sob a premissa do desenvolvimento sustentável;

Portanto, de forma geral, o Gerenciamento de Resíduos Sólidos envolve a aplicação de métodos e tecnologias para implementação dos planos de gerenciamento e, de maneira mais ampla, a Gestão Integrada de Resíduos Sólidos (que envolve outros aspectos relacionados com a gestão). Assim, na definição dos métodos e tecnologias a serem utilizadas no gerenciamento, a gestão integrada deve se embasar nos aspectos legais que regem o setor de resíduos sólidos na região, tanto na esfera municipal, estadual e nacional, pois, a Política Nacional de Resíduos Sólidos anunciou o Plano Nacional de Resíduos Sólidos (PNRS) e exigiu a elaboração dos Planos Estaduais de Resíduos Sólidos (PERS) e dos Planos Municipais ou Intermunicipais de Resíduos Sólidos (PMRS ou PIMRS) como condição para que estados e municípios tenham acesso a recursos da União, ou por ela controlados, para o setor de resíduos sólidos. Esses planos devem ser elaborados para um horizonte de atuação de 20 anos e revisão a cada quatro anos, tendo como conteúdo, entre outros, diagnósticos, proposições de cenários, metas determinadas e programas, projetos e ações para atendimento a essas metas.

No entanto, o artigo 10 da lei incumbe ao Distrito Federal e aos municípios a gestão integrada dos resíduos sólidos gerados nos respectivos territórios, sem prejuízo das competências de controle e fiscalização dos órgãos federais e estaduais do Sistema Nacional do Meio Ambiente (Sisnama), do Sistema Nacional de Vigilância Sanitária (SNVS) e do Sistema Brasileiro de Inspeção de Produtos de Insumos Agropecuários do Sistema Unificado de Atenção à Sanidade Agropecuária (Suasa) bem como da responsabilidade do gerador pelo gerenciamento de resíduos, consoante o estabelecido na lei. 


\subsection{Prioridades na Gestão e no Gerenciamento de Resíduos Sólidos no Brasil}

O artigo $9^{\circ}$ da Lei n ${ }^{\circ} 12.305 / 2010$ observa a ordem de prioridade na gestão e no gerenciamento de resíduos sólidos: não geração, redução, reutilização, reciclagem, tratamento dos resíduos sólidos e disposição final ambientalmente adequada dos rejeitos.

As etapas de não geração e redução dos resíduos sólidos são intrinsecamente relacionadas e têm prioridade para a Gestão e Gerenciamento de Resíduos Sólidos no Brasil, de acordo com a Lei n ${ }^{\circ}$ 12.305/2010. Segundo o Portal dos Resíduos Sólidos (PRS, 2017), o conceito de não geração de resíduos sólidos está intimamente ligado à eficiência em toda a cadeia produtiva e de serviços com o uso de tecnologias modernas e inovadoras. Lembra ainda que "[...] eficiência é a habilidade de fazer o melhor uso de alguma coisa gastando o mínimo possível, podemos aplicar esse princípio aos consumos de energia elétrica, matéria-prima, acessórios, etc., principalmente no setor produtivo". Esgotados os métodos de não geração de resíduos sólidos, o próximo passo é tentar reduzir ao máximo a sua geração. Assim como na não geração, a redução está diretamente ligada a processos de inovação tecnológica em busca de uma melhor eficiência na produção ou serviços, mas também na criação de novos serviços e produtos com tarefas semelhantes, porém, com maior eficiência.

$\mathrm{O}$ artigo $3^{\circ}$ da referida lei define os conceitos de reutilização, reciclagem e disposição final ambientalmente adequada (BRASIL, 2010):

Reutilização: processo de aproveitamento dos resíduos sólidos sem sua transformação biológica, física ou físico-química, observadas as condições e os padrões estabelecidos pelos órgãos competentes do Sisnama e, se couber, do SNVS e do Suasa;

Reciclagem: processo de transformação dos resíduos sólidos que envolve a alteração de suas propriedades físicas, físico-químicas ou biológicas, com vistas à transformação em insumos ou novos produtos, observadas as condições e os padrões estabelecidos pelos órgãos competentes do Sisnama e, se couber, do SNVS e do Suasa;

Disposição final ambientalmente adequada: distribuição ordenada de rejeitos em aterros, observando normas operacionais específicas de modo a evitar danos ou riscos à saúde pública e à segurança e a minimizar os impactos ambientais adversos.

É importante apresentar dois conceitos relacionados à disposição final ambientalmente adequada, apresentados no artigo $3^{\circ}$ da referida lei (BRASIL, 2010):

Rejeitos: resíduos sólidos que, depois de esgotadas todas as possibilidades de tratamento e recuperação por processos tecnológicos disponíveis e economicamente viáveis, não apresentem outra possibilidade que não a disposição final ambientalmente adequada; Destinação final ambientalmente adequada: destinação de resíduos que inclui a reutilização, a reciclagem, a compostagem, a recuperação e o aproveitamento energético ou outras destinações admitidas pelos órgãos competentes do Sisnama, do SNVS e do Suasa, entre elas a disposição final, observando normas operacionais específicas de modo a evitar danos ou riscos à saúde pública e à segurança e a minimizar os impactos ambientais adversos.

O Tratamento de resíduos sólidos, de acordo com o Portal dos Resíduos Sólidos (PRS, 2017), consiste no "[...] uso de tecnologias apropriadas com o objetivo maior de neutralizar as desvantagens da existência de resíduos [...]. Dessa forma podemos denominar de tratamento de resíduos as várias tecnologias existentes desde a reciclagem até a disposição final de rejeitos". 
Pode-se separar as formas de tratamento de resíduos em três grupos (PRS, 2017):

Tratamento Mecânico: No tratamento mecânico são realizados processos físicos geralmente no intuito de separar (usinas de triagem) ou alterar (reciclagem) o tamanho físico dos resíduos. Neste processo não ocorrem reações químicas entre os componentes como nos muitos casos do tratamento térmico.

Tratamento Bioquímico: os processos de tratamento bioquímico mais conhecidos são:

a) Biodigestão: decomposição da matéria orgânica na ausência de oxigênio nos chamados Biodigestores ou Centrais de Biogás.

b) Compostagem: decomposição da matéria orgânica na presença de oxigênio em Usinas de Compostagem.

Tratamento Térmico: Podemos diferenciar cinco principais processos de tratamento térmicos separados em função da temperatura de operação e o meio onde ocorre o processo. São eles:

a) Secagem: retirada de umidade dos resíduos com uso de correntes de ar. Ocorre na presença do ar atmosférico e temperatura ambiente.

b) Pirólise: decomposição da matéria orgânica a altas temperaturas e na ausência total ou quase total de oxigênio. As temperaturas do processo podem variar de 200 a $900^{\circ} \mathrm{C}$.

c) Gaseificação: transformação de matéria orgânica em uma mistura combustível de gases (gás de síntese). Na maioria dos processos não ocorre uma oxidação total da matéria orgânica em temperaturas variando entre 800 e $1600^{\circ} \mathrm{C}$.

d) Incineração: oxidação total da matéria orgânica com auxílio de outros combustíveis a temperaturas variando entre 850 e $1300^{\circ} \mathrm{C}$.

e) Plasma: desintegração da matéria para a formação de gases.

O BNDES (2013) desenvolveu uma pesquisa importante de levantamento e prospecção de processos e tecnologias utilizados no tratamento e destinação final de resíduos sólidos no Brasil e em países do exterior, como EUA e Japão. O trabalho faz um balanço sistemático e comparativo da forma como cada país lida com o seu lixo apontando ainda para as técnicas mais usadas e as inovações disponíveis nesse segmento. A prospecção desenvolvida nesse trabalho permitiu reunir uma diversidade de informações sobre a temática de resíduos sólidos, possibilitando ao leitor conhecer as tendências e oportunidades futuras no ramo. Santos e Santos (2018) também apontam para um cenário de crescimento no número de patentes de tecnologias verdes direcionados à gestão correta de resíduos sólidos, principalmente a partir da Lei 12.203/2010, quando as ações de políticas públicas nesse setor podem ser mais visíveis.

\subsection{Prospecção Tecnológica como Ferramenta de Conhecimento para Inovação}

O estudo prospectivo tem como principal objetivo construir conhecimento que possa vir a servir de base para identificação de oportunidades e orientação na tomada de decisão por diversos atores em investimentos de pesquisa e desenvolvimento (P\&D), podendo revelar adversidades e incertezas sobre o futuro (SANTOS et al., 2004). De acordo com Oliveira (2009), para alcançar resultados satisfatórios por meio da prospecção é importante que o estudo seja conduzido utilizando métodos complementares que se apoiem mutuamente, de modo que sejam sanados os pontos fracos individuais de cada método. 
Coelho (2003) utiliza o termo prospecção como uma ferramenta de mudança que se define como um modo de pensar o futuro baseado na ação e não na pré-determinação. Para Kupfer e Tigre (2004), prospecção tecnológica pode ser entendida como um método para mapear os possíveis aperfeiçoamentos científicos e tecnológicos futuros que possam vir a influenciar no desenvolvimento de diversas áreas como na indústria, na economia ou na sociedade.

Segundo Quintella et al. (2011) por meio da prospecção tecnológica podem ser levantadas e identificadas não apenas as tecnologias existentes, mas também o estágio de maturidade da tecnologia em questão, sua inserção na sociedade, os aspectos das tecnologias concorrentes e as lacunas a serem preenchidas. Em seu trabalho, a pesquisadora realizou mapeamento tecnológico de métodos de análise ópticos para a determinação da estabilidade oxidativa e da massa específica e viscosidade de óleos e biodiesel, e a avaliação da qualidade de biocombustíveis, combustíveis e suas misturas. Para a construção do mapeamento tecnológico foi realizada uma busca de anterioridade na base de dados de patentes European Patent Office (ESPACENET) por meio do uso de palavra-chave.

Essa visão é corroborada por Amparo, Ribeiro e Guarieiro (2012) que afirma que as informações contidas por meio de levantamento de documentos de patentes por meio do seu estado da arte disponível no seu conteúdo mostram-se como um instrumento eficaz para auxiliar à tomada de decisões, pois permite conhecer e identificar tecnologias relevantes, parceiros, concorrentes no mercado, rotas tecnológicas, investimentos, processos, produtos, pesquisa, desenvolvimento, inovação, fusões e aquisições, dentre outras.

Silva et al. (2015) baseados na relevância dos estudos prospectivos, realizaram uma pesquisa de prospecção tecnológica por meio de buscas de patentes nos bancos de dados para apresentar o estado da técnica das tecnologias no tratamento dos resíduos sólidos. As bases de dados utilizadas foram as do banco de patentes do INPI, do Banco Latinoamericano de Patentes (LATIPAT), da World Intellectual Property Organization (WIPO), do ESPACENET e do United States Patent and Trademark Office (USPTO). E por meio desse trabalho foi possível constatar que houve um aumento no volume de resíduos sólidos motivado pelo crescimento da população mundial; esse acréscimo no número populacional ocasionou uma elevação do consumo criando um aumento na demanda de produtos industrializados essa intensificação da produção industrial não gerou apenas uma ampliação na produção de produtos, mas também, elevou o volume do descarte dos resíduos sólidos. Essa externalidade negativa cria como consequência problemas ambientais, sociais e econômicos graves, no entanto, segundo os autores, essa é uma área que tem urgência no desenvolvimento e na expansão de novas tecnologias e mostra-se como um setor oportuno e promissor no avanço de inovações voltadas para coleta e tratamento de resíduos sólidos.

\section{Metodologia}

O trabalho foi desenvolvido em três etapas principais, revisão de literatura, prospecção e tratamento dos dados, descritas a seguir:

Revisão de Literatura: a revisão de literatura foi realizada na busca das definições e das legislações vigentes relacionadas com o Gerenciamento de Resíduos Sólidos, que subsidiaram nas definições dos termos utilizados nas buscas da prospecção, em sites de revistas indexadas 
como Scielo e no Portal de Periódicos Capes, com os descritores "Gerenciamento de Resíduos Sólidos", "Legislação sobre tratamento de resíduos sólidos" e "Prospecção tecnológica".

Prospecção: a prospecção das patentes sobre resíduos sólidos foi realizada no site do INPI, que possui banco de dados de patentes depositadas no Brasil, por meio dos códigos IPC com os códigos de classificação "B09B3/00 (Destruição de lixo sólido ou transformação em algo útil ou inofensivo)" e "C08J11/00 (Recuperação ou reaproveitamento de materiais residuais)".

Tratamento dos dados: as patentes encontradas foram exportadas da base de patentes do INPI para o software Microsoft Excel, no qual os dados foram tratados, com análise de repetições. Depois dessa fase foram gerados gráficos representativos dos dados na tentativa de extrair as informações necessárias às análises.

\section{Resultados e Discussão}

Foram encontradas 577 patentes para o tema Gerenciamento de Resíduos, distribuídos em dois códigos de pesquisa: C08J11-00 (Recuperação ou aproveitamento de materiais residuais), com 84 patentes; e B09B3-00 (Destruição de lixo sólido ou transformação de lixo sólido em algo de útil ou inofensivo), com 493 patentes. A Figura 1 apresenta o número de patentes depositadas ao longo dos anos, apresentado no gráfico com intervalo de cinco anos, no qual, nota-se: i) o baixo número de patentes no intervalo de 1975 a 1990; ii) o comportamento crescente do número de patentes ao longo das décadas; iii) início de uma tendência de aumento do número de patentes a partir do intervalo com início em 2000; iv) o aumento brusco no número de patentes a partir do intervalo com início em 2005; e v) o grande número de patentes no intervalo dee 2010 a 2015. Essas patentes englobam todas as etapas do Gerenciamento de Resíduos Sólidos previstas na Lei n 12.305/2010, da Política Nacional de Resíduos Sólidos, ou seja, não geração, redução, reutilização, reciclagem, tratamento dos resíduos sólidos e disposição final ambientalmente adequada dos rejeitos.

Figura 1 - Gráfico do número de patentes depositadas ao longo dos anos para o tema Gerenciamento de Resíduos Sólidos

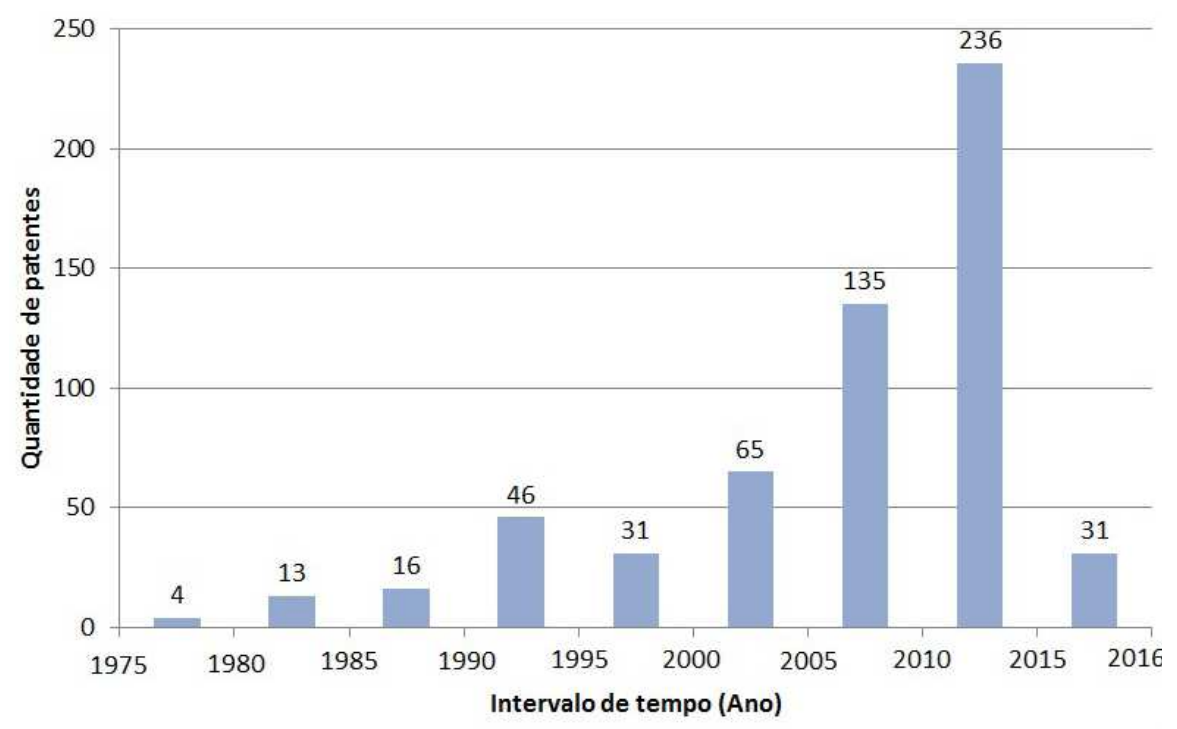

Fonte: Elaborada pelos autores deste artigo (2018) 
A tendência inicial no aumento das patentes, a partir de 2000, assim como o aumento brusco e depois continuado no número de patentes depositadas, a partir de 2010, pode ser justificada pelos movimentos nacionais em torno das discussões relacionadas com os Resíduos Sólidos no Brasil, que culminaram com as criações de duas importantes leis do País (já citadas anteriormente), a Lei 9.795/1999 (BRASIL, 1999), da Política Nacional de Educação Ambiental, e a Lei $n^{\circ}$ 12.305/2010 (BRASIL, 2010), da Política Nacional de Resíduos Sólidos. Nesse caso, as discussões e as novas regulamentações exigidas para as atividades relacionadas com resíduos sólidos devem ter estimulado a educação ambiental e os investimentos em tecnologia e inovação no setor.

A análise das patentes revelou que, dentre as 577 patentes depositadas, apenas $4,7 \%$ (27 patentes, sendo quatro do código C08J11-00 e 23 do código B09B3-00) foram relacionadas com as três primeiras prioridades do Gerenciamento de Resíduos Sólidos no Brasil (Lei $\mathrm{n}^{\circ}$ 12.305/2010), não geração, redução e reutilização, as quais foram o foco do trabalho (Figura 2). Esses resultados mostraram que a grande maioria das patentes depositadas $(95,3 \%)$ está relacionada às etapas de pós-geração de resíduos sólidos, como reciclagem, tratamento dos resíduos sólidos e disposição final ambientalmente adequada dos rejeitos. A Figura 3 mostra o número de patentes depositadas ao longo dos anos para as três etapas prioritárias do Gerenciamento de Resíduos Sólidos, no qual, nota-se que a grande quantidade de patentes depositadas a partir de 2010, a que pode ser justificada de maneira semelhante à justificativa dada anteriormente à variação de patentes total.

Figura 2 - Gráfico ilustrativo da relação (porcentagem) entre a quantidade de patentes solicitadas para todas as etapas do Gerenciamento de Resíduos Sólidos (em vermelho) e as etapas prioritárias de não geração, redução e aproveitamento (em azul)

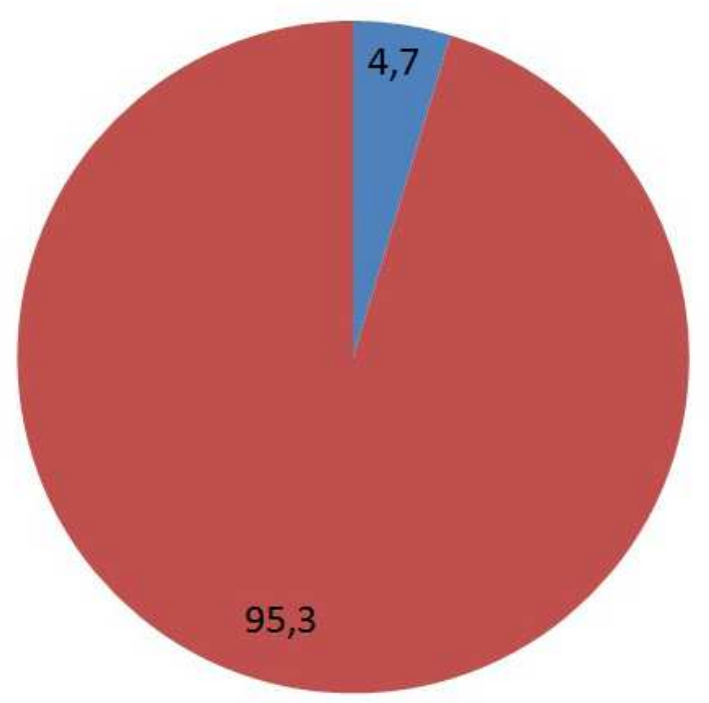

Fonte: Elaborada pelos autores deste artigo (2018) 
Figura 3 - Gráfico do número de patentes depositadas ao longo dos anos para as etapas prioritárias no Gerenciamento de Resíduos Sólidos: não geração, redução e reutilização

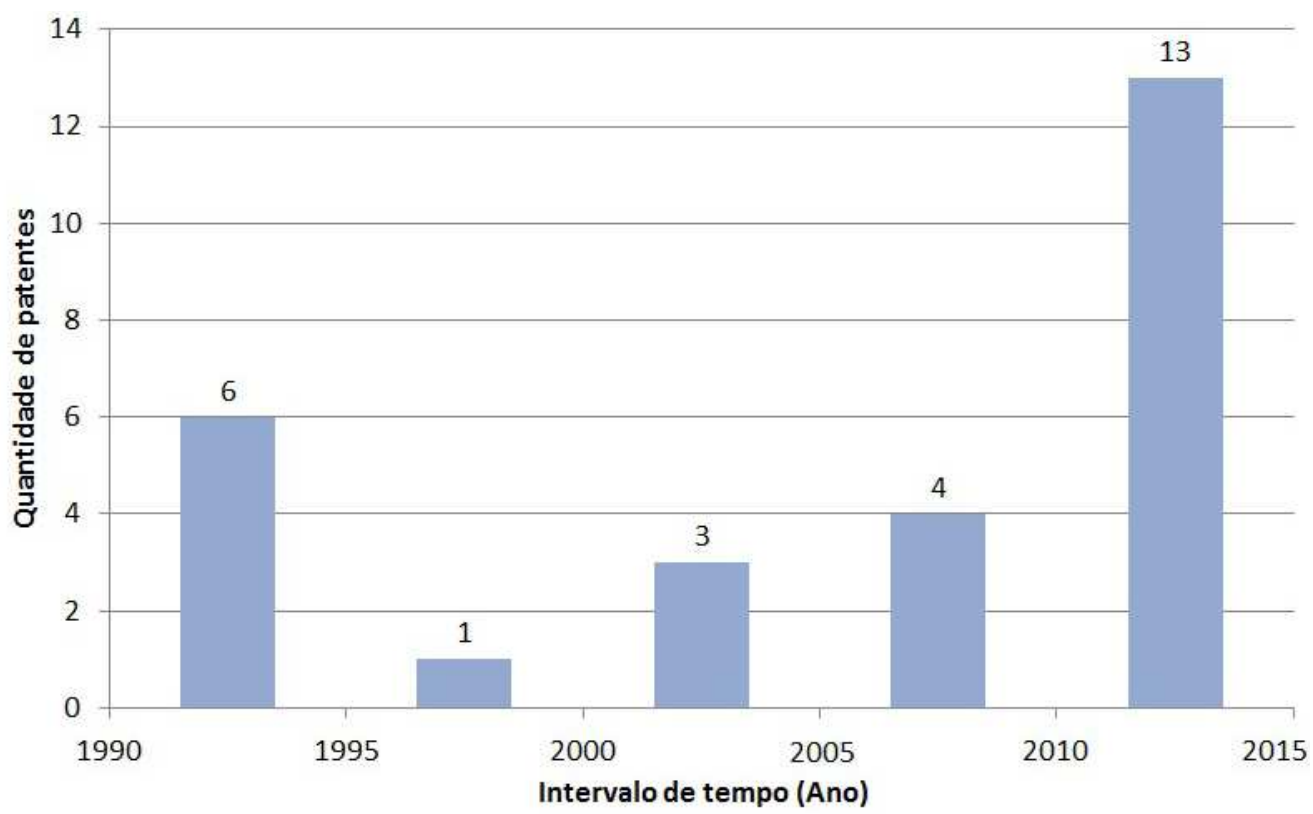

Fonte: Elaborada pelos autores deste artigo (2018)

A análise mais detalhada das patentes relacionadas com as prioridades citadas anteriormente (Figura 2) não permitiu indicação de tendências tecnológicas ao longo dos anos, com os temas variando desde: "processo para valorização de resíduos sólidos, principalmente de lixo doméstico e dispositivo para sua operação", "método e aparelho para redução controlada de material orgânico", e "processo de aproveitamento de resíduos de porcos" no início da década de 1990; passando na década de 2000 por "máquina coletora de latas de alumínio com prêmios sorteados", "método para reutilização de resíduos industriais sólidos provenientes de madeira para uso como energia", e "processo para reaproveitamento de papelão e produto obtido"; até "revestimento reutilizando telhas de cerâmica", "processo de reaproveitamento de resíduos descartados em aterros sanitários", e "equipamento para secagem de resíduos domésticos" a partir de 2010.

No entanto, nos últimos 10 anos, já se observou patentes relacionadas com as questões do "lixo eletrônico", como "método para descartar equipamento elétrico e eletrônico", em 2009, "método e dispositivo para desmontagem de aparelhos elétricos e ou eletrônicos, em 2010", "processo e dispositivo para fragmentação de aparelhos de refrigeração, em 2011", e "processo para reaproveitamento de resíduo de toner e produto resultante", em 2015.

\section{Considerações Finais}

A prospecção tecnológica mostrou que, apesar de a Lei n $12.305 / 2010$, da Política Nacional de Resíduos Sólidos, destacar as etapas de não geração, redução e reutilização como prioritárias para o Gerenciamento de Resíduos Sólidos no Brasil, apenas 4,7\% das 577 patentes depositadas sobre o tema foram relacionadas a essas etapas. Isso indica pouco investimento no setor, apesar das campanhas "lixo zero" criadas em vários estados da Federação. Contudo, campanhas ocorridas recentemente no Brasil, assim como uma maior disseminação da legislação 
e uma educação ambiental mais eficiente e transformadora, poderão tornar o setor de resíduos sólidos promissor para investimentos de pesquisa, desenvolvimento e apropriação tecnológica.

Espera-se que este trabalho contribua para despertar o interesse dos pesquisadores brasileiros na pesquisa aplicada na área de resíduos sólidos, principalmente quanto a não geração de resíduos sólidos, para que haja desenvolvimentos científicos e tecnológicos futuros, incentivando a geração de patentes. Como continuidade do trabalho, serão realizadas análises qualitativas com relação às patentes solicitadas, assim como a análise das principais redes de relacionamento entre inventores de patentes, suas parcerias e afiliações.

\section{Referências}

AMPARO, K. K. dos S.; RIBEIRO, M. do C. O.; GUARIEIRO, L. L. N. Estudo de caso utilizando mapeamento de prospecção tecnológica como principal ferramenta de busca científica.

Perspectivas em Ciência da Informação, Belo Horizonte, v. 17, n. 4, p. 195-209, 2012.

BANCO NACIONAL DE DESENVOLVIMENTO ECONÔMICO E SOCIAL (BNDES). Análise das diversas Tecnologias de Tratamento e Disposição Final de Resíduos Sólidos no Brasil, Europa, Estados Unidos e Japão. FADE - UFPE. 2013. Disponível em: < http://www.abetre.org. br/estudos-e-publicacoes/publicacoes/publicacoes-abetre/UFPEFADETecnologiasparaRSU2013.pdf>. Acesso em: 8 jun. 2018.

BRASIL. Lei no 9.795, de 27 de abril de 1999. Dispõe sobre a educação ambiental e institui a Política Nacional de Educação Ambiental. 1999. Disponível em: <www.planalto.gov.br/ccivil_03/leis/ L9795.htm > Acesso em: $1^{\circ}$ jul. 2017.

Lei $n^{\circ}$ 12.305, de 2 de agosto de 2010. Institui a Política Nacional de Resíduos Sólidos; altera a Lei $\mathrm{n}^{\circ}$ 9.605, de 12 de fevereiro de 1998; e dá outras providências.. 2010. Disponível em: <www.planalto.gov.br/ccivil_03/_ato2007-2010/2010/lei/112305.htm> Acesso em: 10 jul. 2017.

COELHO, G. M. Prospecção tecnológica: metodologias e experiências nacionais e internacionais. Nota Tecnica 14. Projeto CTPETRO. Rio de Janeiro: Instituto Nacional de Tecnologia, 2003.

INSTITUTO NACIONAL DE PROPRIEDADE INTELECTUAL (INPI). [Base de dados - Internet]. 2017. Disponível em: <www.inpi.gov.br/> Acesso em: $1^{\circ}$ jul. 2017.

KUPFER, D.; TIGRE, P. B. Modelo SENAI de Prospecção: Documento Metodológico. Capítulo 2: Prospecção Tecnológica. In: Organizacion Internacional del Trabajo Cinterfor. Montevideo: OIT/ CINTERFOR; 2004. Papeles de la Oficina Técnica n. 14.

OLiVeirA, M. M. M. A Prospecção Tecnológica como Ferramenta de Planejamento Estratégico para a Construção do Futuro do Instituto Oswaldo Cruz. 2009. 103 f. Dissertação (Mestrado em Saúde Pública) - Escola Nacional de Saúde Pública Sergio Arouca. Fundação Oswaldo Cruz, Brasília, DF, 2009.

PORTAL DOS RESÍDUOS SÓLIDOS (PRS). 2017. Disponível em: <http://www. portalresiduossolidos.com >. Acesso em: $1^{\circ}$ jul. 2017.

QUINTELLA, C. M. et al. Prospecção Tecnológica como uma Ferramenta Aplicada em Ciência e Tecnologia para se Chegar à Inovação. Revista Virtual de Química, Rio de Janeiro, v. 3, n. 5, p. 406-415, 2011. 
SANTOS, C. S. F. et al. Prospecção de tecnologias de futuro: métodos, técnicas e abordagens.

Parcerias Estratégicas, [S.1.], v. 9, n. 19, 2004. Disponível em: <http://seer.cgee.org.br/index.php/ parcerias_estrategicas/article/view/253>. Acesso em: 5 jul. 2018.

SANTOS, N. J. B. dos; SANTOS, M. J. C. dos. Mapeamento do desenvolvimento tecnológico de patentes verdes relacionadas ao gerenciamento de resíduos. Caderno Prospecção, Salvador, v. 11, n. 1, p. 17-25, jan./mar. 2018.

SILVA, J. B. et al. Prospecção tecnológica no tratamento de resíduos sólidos. In: ENCONTRO INTERNACIONAL SOBRE GESTÃO EMPRESARIAL E MEIO AMBIENTE. Engema XVII, 2015, São Paulo. Anais... São Paulo: Faculdade de Economia, Administração e Contabilidade da USP. Disponível em: <http://engemausp.submissao.com.br/17/anais/arquivos/61.pdf> Acesso em: 6 jul. 2018.

\section{Sobre os autores}

\section{Laudilene Macedo Bispo}

E-mail: lenebispo@yahoo.com.br

Mestranda do Mestrado Profissional em Propriedade Intelectual e Transferência de Tecnologias para a Inovação na Universidade Estadual de Santa Cruz (UESC); graduada em História, pela UESC; especialista em Pedagogia Empresarial Estratégica, pela Universidade Potiguar (UNP); especialista em Sociedade, Inovação e Tecnologia Social, pela Universidade Federal do Recôncavo da Bahia (UFRB); especialista em Gestão Pública, pela Universidade Católica Dom Bosco (UCDB).

Endereço profissional: Universidade Estadual De Santa Cruz. Campus Soane Nazaré De Andrade, Rodov. Jorge Amado, Km 16 - Salobrinho, Ilhéu, BA.

\section{Josiele Ferreira do Nascimento}

E-mail: jfnascimento@uesc.br

Graduada em Ciências Econômicas, pela Universidade Estadual de Santa Cruz (UESC). Mestranda PROFNIT UESC.

Endereço profissional: Universidade Estadual De Santa Cruz. Campus Soane Nazaré De Andrade, Rodov. Jorge Amado, Km 16 - Salobrinho, Ilhéu, BA.

\section{Marcelo Soares Teles Santos}

E-mail: marcelostsantos@gmail.com

Doutor em Ciência e Engenharia de Petróleo, pela Universidade Federal do Rio Grande do Norte (UFRN); mestre em Geofísica, pela Universidade de São Paulo (USP); e graduado em Engenharia de Agrimensura, pela Universidade Federal de Viçosa (UFV).

Endereço profissional: Universidade Federal do Sul da Bahia. Rodovia de Acesso para Itabuna, km 39 - Ferradas, Itabuna, BA. CEP: 45613-204. 